\title{
Segmentation of Aerial Images for Plausible Detail Synthesis
}

\author{
Oscar Argudo ${ }^{\mathrm{a}}$, Marc Comino $^{\mathrm{a}}$, Antonio Chica $^{\mathrm{a}}$, Carlos Andújar $^{\mathrm{a}}$, Felipe Lumbreras $^{\mathrm{b}}$ \\ ${ }^{a}$ ViRVIG, Computer Science Department, Universitat Politècnica de Catalunya \\ Jordi Girona 1-3, Barcelona, Spain \\ ${ }^{b}$ Computer Vision Center, Department of Computer Science, Universitat Autonòma de Barcelona \\ Edifici $O$, Bellaterra, Spain
}

\begin{abstract}
The visual enrichment of digital terrain models with plausible synthetic detail requires the segmentation of aerial images into a suitable collection of categories. In this paper we present a complete pipeline for segmenting high-resolution aerial images into a user-defined set of categories distinguishing e.g. terrain, sand, snow, water, and different types of vegetation. This segmentationfor-synthesis problem implies that per-pixel categories must be established according to the algorithms chosen for rendering the synthetic detail. This precludes the definition of a universal set of labels and hinders the construction of large training sets. Since artists might choose to add new categories on the fly, the whole pipeline must be robust against unbalanced datasets, and fast on both training and inference. Under these constraints, we analyze the contribution of common per-pixel descriptors, and compare the performance of state-of-the-art supervised learning algorithms. We report the findings of two user studies. The first one was conducted to analyze human accuracy when manually labeling aerial images. The second user study compares detailed terrains built using different segmentation strategies, including official land cover maps. These studies demonstrate that our approach can be used to turn digital elevation models into fully-featured, detailed terrains with minimal authoring efforts.
\end{abstract}

Keywords: Terrain editing, Detail synthesis, Vegetation synthesis, Terrain rendering, Image segmentation

\section{Introduction}

2 Publicly available Digital Terrain Models (DTM) and aerial ${ }_{3}$ images have opened new possibilities for using real scenarios in 4 video games and entertainment applications. These $2.5 \mathrm{D}$ mod${ }_{5}$ els are readily usable for rendering aerial views of the scenes, ${ }_{6}$ but lack resolution and 3D appearance for close-up views. As a 7 consequence, artists often need to enrich DTMs with synthetic 8 detail, such as procedural bedrock and rocks for the ground, 9 realistic water shaders for lakes and rivers, and fully-detailed 10 plant models for the vegetation. When done manually, a sub${ }_{11}$ stantial amount of effort is required to locate the different ele12 ments in the aerial images and to apply a suitable detail synthe13 sis technique to them.

14 In this paper we address the problem of segmenting high${ }_{15}$ resolution $(25 \mathrm{~cm} /$ pixel $)$ aerial images into small sets of classes ${ }_{16}$ suitable for detail synthesis (Figures 1 and 2). The segmenta17 tion for synthesis problem ( $S 4 S$ from now on) exhibits a num${ }_{18}$ ber of unique issues that we summarise below. First, categories ${ }_{19}$ in S4S are defined according to the different detail synthesis 20 techniques artists might want to apply. For example, in a par${ }_{21}$ ticular desert scene for a Dakar rally game, one artist might 22 want to distinguish rock, sand, cacti and palm trees, whereas ${ }_{23}$ in a tropical forest scene we could be interested in segmenting 24 vegetation and rivers.

25 Second, we want to give artists the possibility to add new ${ }_{26}$ classes dynamically. This way artists can progressively refine ${ }_{27}$ the appearance of different materials, which due to their variety 28 are hard to know in advance (e.g. forest, shrub, grass, crops,
29 sand, bare rock, scree, water courses, inland marshes, snow...) 30 and can decide to distinguish non-anticipated categories (e.g. ${ }_{31}$ deciduous forest from coniferous forest). This flexibility means 32 that the pixel classifier should be able to work with relatively зз small training sets (containing examples from a varying set of ${ }_{34}$ classes) and that both training and classification times should be 35 within the range of a few minutes. Notice also that we cannot ${ }_{36}$ assume balanced classes in the segmented exemplars, neither ${ }_{37}$ the exemplar class distributions to be representative of the true 38 class distributions.

39 In this context, generating large and varied training sets is 40 unfeasible. Manual image segmentation requires a substantial ${ }_{41}$ amount of effort. For example, a $25 \mathrm{~cm} /$ pixel image covering ${ }_{42} 1 \mathrm{~km}^{2}$ contains $16 \mathrm{M}$ pixels. Even when using the advanced ${ }_{43}$ tools found in state-of-the-art object-based image processing 44 applications (multiresolution segmentation, superpixels, man${ }_{45}$ ual relabeling...), complete segmentation of a $1 \mathrm{~km}^{2}$ image can 46 take several hours of an expert human classifier. Moreover, we 47 will show that resulting labels largely depend on the labeler's 48 judgement and thus cannot be safely taken as ground truth.

49 This manual labeling effort can be largely alleviated by seg50 menting only a collection of easy-to-label uniform regions (see ${ }_{51}$ Figure 9) and taking the training examples from these regions ${ }_{52}$ using a suitable sampling strategy. We adopt this approach (par${ }_{53}$ tial, region-based classification) as the only feasible approach in 54 the context of dynamic classes.

${ }_{55}$ Although there is an extensive literature on image segmen${ }_{56}$ tation in remote sensing, previous approaches either require 

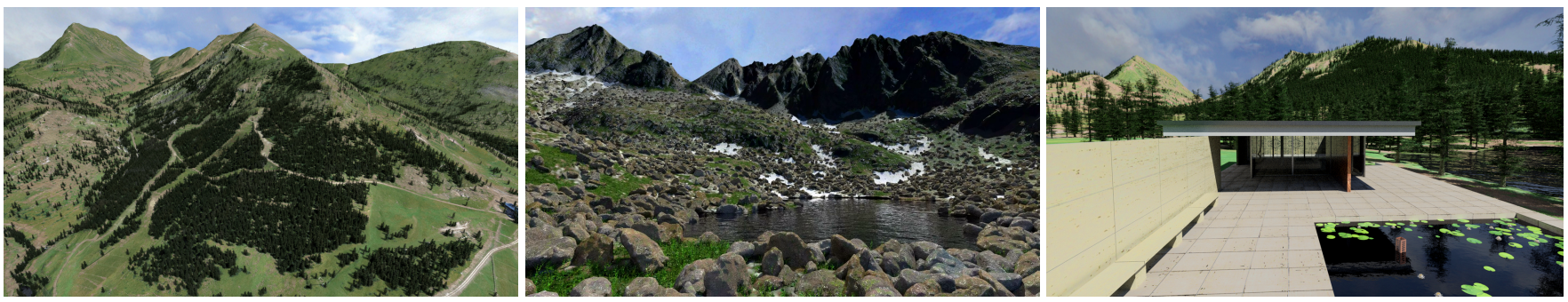

Figure 1: Renders of detailed terrains created from aerial images. The segmentation of the aerial image allows for the application of different techniques depending on the soil cover. In these examples, synthetic trees, bushes and grass for vegetation areas, fractal displacement for the bare soil, rocks and gravel shader for unpaved roads, and specific shaders for snow, lakes and rivers. The last image shows an example of landscape and visual impact assessment.

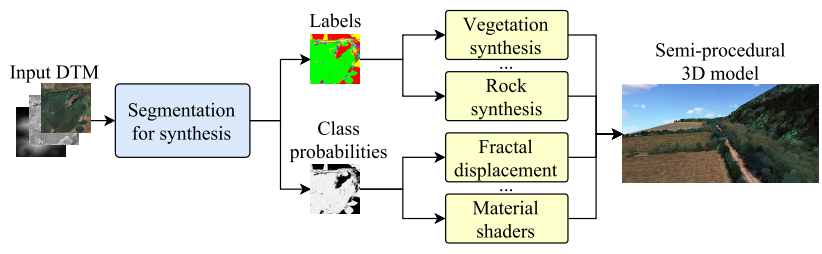

Figure 2: We address the problem of adding realism to digital terrain models through the segmentation of aerial images (blue box) into a suitable set of classes (e.g. vegetation, rock, water). This way each class can be rendered using specific shaders and procedural content (yellow boxes).

${ }_{57}$ extensive training sets (unfeasible in the context of dynamic ${ }_{58}$ categories), use descriptors tuned for very specific categories 59 (e.g. crops), assume balanced, representative datasets, or rely 60 on expensive-to-train classification algorithms.

${ }_{61}$ To the best of our knowledge, this is the first work explor${ }_{62}$ ing the optimal components of a standard image segmentation ${ }_{63}$ pipeline specifically tailored for segmentation-for-synthesis.

64 The key contributions of the paper are:

- A complete pipeline for training and inferring per-pixel labels from a dynamic, user-defined set.

- A performance comparison of state-of-the-art machine learning algorithms for S4S.

- An analysis on the contribution of different pixel descriptors (at varying resolution levels) in the classifier accuracy.

- A discussion on different strategies for sampling the training set from partially-segmented exemplars.

- A user study analyzing human accuracy when manually labeling uniform regions in aerial images. We estimate the difficulty of the regions, the expertise of the labelers, and the true label of the regions.

- A second user study demonstrating the effectiveness of our approach. We asked users to compare renders built from images segmented using either our approach or official land cover maps.

\section{${ }_{81}$ 2. Previous work}

${ }_{82}$ Despite the specific nature of the S4S problem discussed sз above, we rely on the great amount of work already done in 84 the field of aerial image analysis and on typical strategies of 85 region analysis based on color and texture.

${ }_{86}$ Classical image segmentation techniques compute per-pixel 87 features based on image values in the pixel's neighborhood. ${ }_{88}$ Many different pixel descriptors have been proposed both for ${ }_{89}$ general images and remote sensing images. Ruiz et al. [1] 90 compared different texture and spectral feature descriptors for ${ }_{91}$ pixel classification of remote sensing images. Results on differ${ }_{92}$ ent forest scenes showed that there is no universal criteria - the ${ }_{93}$ suitable set of features depends on the type of landscape units ${ }_{94}$ defined in each application. Similarly, dos Santos et al. [2] also ${ }_{95}$ compared the effectiveness of various color and texture descrip${ }_{96}$ tors for image classification. Although their task was not pixel ${ }_{97}$ based, the best descriptors were also dependant on the type of ${ }_{98}$ input dataset. Tokarczyk et al. [3] compare classical feature 99 sets with feature banks computed with the first layers of deep 100 networks. Their results show that features based on patches 101 dominate over those based on individual pixels, i.e. texture 102 holds important information in high-resolution images. How103 ever, complex feature extraction methods or even non-linear 104 feature learning yield small or no improvement, while adding a ${ }_{105}$ significant computation cost. Penatti and dos Santos [4] studied 106 whether Convolutional Neural Networks trained for classifica107 tion of everyday objects generalize to aerial and remote sensing 108 images. They used the output of the last fully-connected layer 109 of two networks (OverFeat and CaffeNet) as features, and com110 pared with low-level feature descriptors. On the aerial dataset, ${ }_{111}$ deep features achieved the best results, but for remote sensing 112 images they were outperformed by the low-level descriptors. ${ }_{113}$ Since we do not know in advance the specific classes artists 114 will require, and training sets are expected to be too small for 115 end-to-end learning, we use a large number of color and texture 116 features from the literature, combined with height, slope and 117 gradient descriptors from the DTM.

${ }_{118}$ Multiple works also compare the accuracy of classifica${ }_{119}$ tion algorithms in the context of segmentation of aerial im120 ages, including Bayesian classifiers [5], Random Forests [3], ${ }_{121}$ SVM [6, 4] and Deep Learning methods [7]. Frölich et al. pre122 sented Iterative Context Forests [8, 9], a classification system 
${ }_{123}$ based on Random Forests that builds the trees level-wise and 124 adds the class probability maps of one level as a new input 125 channel for the next level. A similar approach by Tokarczyk 126 et al. [10] uses boosting instead of Random Forests. Castelluc${ }_{127}$ cio et al. [7] compared three different options for classification 128 with convolutional neural networks: training from scratch, fine129 tuning the last layers using training images, or using the last ${ }_{130}$ fully-connected layer as features as in [4]. On the aerial images ${ }_{131}$ dataset, the best accuracies were achieved by fine-tuning, then ${ }_{132}$ using the feature vector, and finally by training from scratch. ${ }_{13}$ On the remote sensing dataset, training from scratch or fine134 tuning provided the best results depending on the network used. ${ }_{135}$ The authors report that training from scratch the $\mathrm{CNN}$ took days ${ }_{136}$ or weeks of computation time.

${ }_{137}$ In contrast to the previous techniques, our approach focuses ${ }_{138}$ on obtaining segmentations that will be used for detail synthe139 sis. Segmentation classes are dynamically defined by the user, 140 which requires fast training and inference times and prevents 141 the use of massive training sets. Unfortunately, many works 142 in the literature do not focus on inference and training times. ${ }_{143}$ We compare different state-of-the-art classification algorithms 144 by considering all these criteria and the robustness against un145 balanced data, as well as the ability to generate per-class prob146 ability maps (besides class labels).

147 Keeping the class with maximum a posteriori probability as 148 the final pixel class can result in noisy labelings. The smooth149 ness assumption states that nearby pixels tend to have similar 150 labels, thus individual pixels are not independent variables but 151 form a random field. Schindler [11] compared different smooth152 ing strategies that increase accuracy: majority voting in a neigh153 borhood, Gaussian and bilateral filtering, and approximate in154 ference via global methods for random fields such as graph cuts 155 and semiglobal labeling. They also note that including fea156 ture descriptors computed on the pixel neighbourhood implic157 itly produces smoother labelings. We propose a variation of the 158 bilateral filter exploiting domain knowledge on the classes.

\section{3. Overview}

160 Given a small collection of segmented exemplars $E$, and an ${ }_{161}$ input aerial image $I$ of a non-urban area, we aim to classify each 162 pixel of $I$ into a user-defined set of classes including e.g. bare ${ }_{163}$ soil and different vegetation types. Figure 3 shows an overview ${ }_{164}$ of the complete pipeline. Note that this is a typical supervised ${ }_{165}$ classification pipeline. Our contribution though is the analysis 166 of the algorithms for feature extraction, sampling, classifica167 tion, smoothing, and cost-aware labeling, that achieve the best 168 trade-off between training set size, running times, and accuracy, 169 in the context of dynamic labels.

170 All images (exemplars and images to be segmented) are as171 sumed to be high-resolution $(25 \mathrm{~cm}$ per pixel), orthorectified 172 aerial images providing reflected light measurements in the red ${ }_{173}(\mathrm{R})$, green $(\mathrm{G})$, blue (B) and near-infrared (I) bands of the elec174 tromagnetic spectrum. This kind of aerial images are often pub175 licly available through Web Map Services [12]. We did not con176 sider hyperspectral images [13] employing a larger number of 177 spectral bands because these images are not publicly available
178 except for specific areas and at lower resolutions. We also as179 sume an elevation map of the region is also available (we used $1805 \mathrm{~m}$ per pixel elevation data from public Web Coverage Ser181 vices).

${ }_{182}$ The output of the algorithm is the segmented image, with ${ }_{183}$ each pixel classified into one of the user-provided classes, along 184 with the associated class probability maps (one per class). Seg185 mented regions can be used to control the instancing of pro${ }_{186}$ cedural 3D content (e.g. rocks, tree, grass) whereas probabil187 ity maps are useful to get smooth transitions across material 188 boundaries and to weight the influence of material shaders.

All our sets include at least the following labels: bare ground, 190 tree/shrub and grass. The choice of these classes is motivated 191 by their prominence in typical terrains, and the clearly differ192 ent CPU/GPU techniques required for their detailed represen193 tation. Terrain regions can be enhanced e.g. through fractal 194 noise [14, 15, 16], by adding bedrocks and rock layers [17], and 195 by synthesizing high resolution detail through multi-resolution 196 dictionary-based approaches $[18,19]$. Both trees and shrubs 197 are often rendered as billboard clouds [20] whereas grass is of198 ten handled through specific rendering techniques [21, 22].

The training part of the pipeline is shown in Figure 3. A 200 few exemplars are labeled manually, we extract a collection 201 of features for each pixel (Section 4), and train a multi-class 202 classifier on a subset of segmented pixels. As we shall see, 203 we found Random Forest (RF) to be the best option consid204 ering accuracy and performance both at training and inference 205 time (Section 5). The user may at any moment add new classes 206 and provide new segmented areas in the existing exemplars, or 207 add new exemplars containing representative areas from these 208 classes. Adding new labels obviously involves retraining the 209 classifier, but not repeating the feature extraction for existing 210 exemplars. Since exemplars are selected and segmented by the 211 user, we cannot assume classes to be balanced, and we cannot 212 either suppose their distribution to be representative of the true ${ }_{213}$ class distribution. This means dataset sampling [23] might play 214 a key role in accuracy.

215 For labeling automatically the input aerial images, we use ${ }_{216}$ the trained RF to predict class probabilities. Although per-pixel 217 features are computed on a neighborhood around the pixel, at 218 this stage class probabilities are often noisy. We thus apply 219 a smoothing step (a bilateral filter) to each of the individual 220 class probabilities (Section 8). Finally, we allow the user to 221 apply a cost matrix to choose as final label the one minimizing 222 perceptual misclassification costs (Section 9).

\section{${ }_{223}$ 4. Tested features}

224 As stated above, previous studies on the segmentation of 225 aerial images have found that the optimal feature set depends 226 on the specific set of categories [1, 2]. Recent deep learning ap${ }_{227}$ proaches obviate feature engineering, but for the specific case 228 of pixel-wise aerial image segmentation they have shown lit229 tle or no improvement with respect to low-level features [3, 4]. ${ }_{230}$ Since we want to let artists choose the desired classes, we de${ }_{231}$ cided to include a large number of well-known color and tex232 ture features from the literature, and test their contribution on 


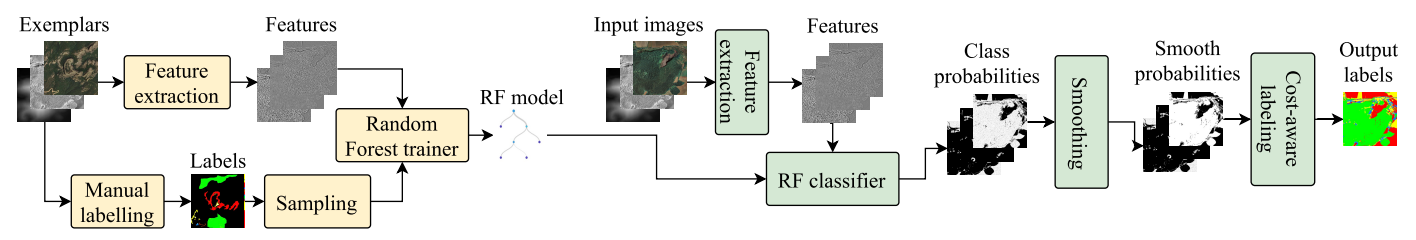

Figure 3: Overview of the segmentation pipeline. Training steps are shown in orange and inference steps in green.

${ }_{233}$ the classification of typical classes (Section 6). For the sake of 234 reproducibility, we list below the 91 features we tested. Please 235 refer to $[1,24,25]$ for further details. Features were computed 236 based on the red, green, blue, near infrared and elevation of the ${ }_{237}$ pixel itself and its neighborhood, at the original resolution of 238 the input images $(25 \mathrm{~cm} /$ pixel $)$. An analysis of the features at 239 lower resolutions is provided in Section 6.

240 Height features [4 values]: we considered the elevation at 241 the pixel location as well as its gradient (orientation and mag242 nitude). Orientation is represented with two $\sin / \cos$ values to ${ }_{243}$ avoid discontinuities. As we shall see, we propose to use gra244 dient magnitude but discard elevation and gradient orientation 245 (see Section 6).

246 Color features [10 values]: we use the RGB color compo247 nents of the pixel, as well as its components in HSL and CIELab 248 color spaces. Hue is represented with $\sin / \cos$ values.

249 NDVI [1 value]: the Normalized Difference Vegetation In250 dex [26] is the ratio $(I-R) /(I+R)$ where $I$ and $R$ represent the 251 infrared and red values.

252 GLCM [20 values]: We compute four normalized Grey ${ }_{253}$ Level Co-occurrence Matrices on $15 \times 15$ windows with dis${ }_{254}$ placement vectors $(0,1),(1,0),(1,1)$ and $(-1,1)$. As in [24], 255 we extract from each matrix five features: energy, entropy, con256 trast, homogeneity and correlation.

257 Spectral features [6 values]: The power spectrum of natu258 ral images tends to follow the power law $P=1 / f^{\beta}$, where $P$ 259 is the power as a function of frequency $f$ and $\beta$ is the spectral 260 slope [25]. We compute $P$ and $\beta$ on $16 \times 16$ windows. We com261 puted four extra features by dividing the frequency domain into ${ }_{262} 4$ ring-shaped regions, and computing the power sum on each 263 region.

HOG features [8 values]: the Histogram of Oriented Gra265 dients was computed on a window of 15 pixels with lightness 266 grouped into 8 bins.

267 LBP [42 values]: we compute a rotationally invariant ver268 sion of the Local Binary Pattern descriptor [27] over the HSL 269 and RGB channels, using 8 points and radii 1, 2, 4 and 8, and 270 using 16 points and radii 16,32 and 64 .

\section{5. Comparison of classification algorithms for $\mathrm{S} 4 \mathrm{~S}$}

272 We compared the performance of multiple traditional classi273 fication algorithms, aiming to obtain the best possible accuracy 274 while achieving interactive training and inference times. We 275 tested the K Nearest Neighbours algorithm with $k=1,10$ 276 and 20 neighbours, Multinomial Regression with and with277 out normalized features and Random Forests with $N=100$
278 trees and $D \leq 15$ maximum depth. For these tests we aver279 aged execution times over 5 runs. Finally we also tested SVMs 280 with linear (gamma and bias set to 1$)$ and $\operatorname{RBF}(\sigma=1)$ kernels. 281 Results are reported in Table 1.

\begin{tabular}{rrrr}
\hline Algorithm & Accuracy & Training & Inference \\
\hline Random Forest (N=50, D $\leq 15)$ & $\mathbf{9 4 . 8 0 \%}$ & $6 \mathrm{~min} 9 \mathrm{~s}$ & $58 \mathrm{~s}$ \\
SVM Polynomial (linear) & $93.80 \%$ & $13 \mathrm{~h} 26 \mathrm{~min}$ & $3 \mathrm{~h} 7 \mathrm{~min}$ \\
SVM RBF $(\sigma=1.0)$ & $\mathbf{9 4 . 6 8 \%}$ & $5 \mathrm{~h} 35 \mathrm{~min}$ & $2 \mathrm{~h} 59 \mathrm{~min}$ \\
Logistic Regression & $93.78 \%$ & $1 \mathrm{~h} 40 \mathrm{~min}$ & $1 \mathrm{~min} 42 \mathrm{~s}$ \\
Logistic Regression (norm.) & $93.76 \%$ & $1 \mathrm{~h} 36 \mathrm{~min}$ & $45 \mathrm{~s}$ \\
kNN $(\mathrm{k}=20$, norm.) & $92.46 \%$ & $\mathrm{n} / \mathrm{a}$ & $3 \mathrm{~h} 52 \mathrm{~min}$ \\
kNN (k=10, norm.) & $92.51 \%$ & $\mathrm{n} / \mathrm{a}$ & $3 \mathrm{~h} 36 \mathrm{~min}$ \\
$\mathrm{kNN}(\mathrm{k}=1$, norm.) & $90.44 \%$ & $\mathrm{n} / \mathrm{a}$ & $2 \mathrm{~h} 59 \mathrm{~min}$ \\
\hline
\end{tabular}

Table 1: Average times and accuracies of the different machine learning algorithms we tried. In some cases features were normalized (indicated as norm).

282 Random Forest, SVM and Logistic Regression all provided 283 similar accuracies. However, Random Forest was much faster 284 to train than the other two, also offering the fastest classification 285 times. We thus claim that Random Forest is a very good option 286 for our purposes.

${ }_{287}$ Random Forests can be tuned using two main parameters: the 288 depth (D) of the trees and number $(\mathrm{N})$ of trees. For each com289 bination of $D \in\{5,10,15,25\}$ and $N \in\{10,25,50,75,100\}$, we 290 measured the average accuracy obtained on 10 different pairs 291 of training and validation sets from Dataset Andorra. Figure 4 292 shows the observed accuracies. Unless explicitly stated, all ex293 periments in this article use $N=50$ and $D \leq 15$ since these 294 values give the best trade-off between performance and accu295 racy. Our results are similar to the ones obtained by [3], who 296 also chose $N=50, D \leq 15$. Regarding the number of fea297 tures each tree can randomly select, all tests were set up to use $298\left\lceil\log _{2} 91\right\rceil=7$ features.

299 Since Deep Learning approaches do not use the features dis300 cussed above, we discuss Convolutional Neural Networks sep301 arately (Section 11).

\section{${ }_{302} 6$. Feature analysis}

\section{6.1. Multiresolution features}

304 Some features in the chosen feature set are computed us305 ing sliding windows of up to 16 pixels (except LBP, which 306 uses larger radii). Since our input data has a resolution of $30725 \mathrm{~cm} /$ pixel, our feature set takes into account neighbourhoods 308 up to $4 \mathrm{~m}$ wide. We could increase this span either by increas309 ing the sliding window size - which would also significantly 


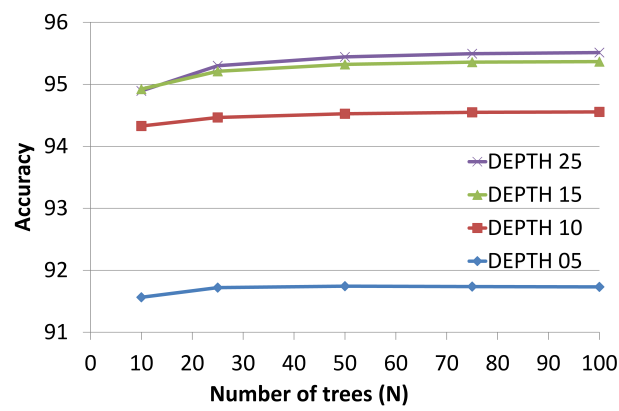

Figure 4: Accuracy of Random Forests depending on number of trees $(\mathrm{N})$ and maximum depth (D) on Dataset Andorra.

310 increase feature extraction costs -, or using an image of the ${ }_{311}$ same area at a coarser resolution.

${ }_{312}$ We performed an experiment using again Dataset Andorra ${ }_{313}$ to assess whether this multiresolution approach improves ac314 curacy. We used a training set and a validation set, each one 315 containing about $2 \%$ of the input image pixels (about $260 \mathrm{~K}$ 316 samples), and measured the accuracy of a RF classifier with 317 features computed at varying resolution levels. We averaged 318 the results over 10 executions.

319 Our baseline performance was given by a minimum feature 320 set which included the four height features $\mathrm{H}$ (elevation, gra${ }_{321}$ dient magnitude and orientation), as well as the LBP features. $322 \mathrm{LBP}$ was included in the baseline because it is computed using ${ }_{323}$ radii up to 64 pixels. We then added the rest of the features 324 (F) computed at varying resolutions: $25 \mathrm{~cm} /$ pixel, $50 \mathrm{~cm} /$ pixel, $3251 \mathrm{~m} /$ pixel and $2 \mathrm{~m} /$ pixel.

Table 2 shows the resulting accuracies. Since the best accura327 cies were given by the highest resolution image $(25 \mathrm{~cm} / \mathrm{pixel})$, 328 we also tried combining the features of this one with one of 329 the downsampled images. Results slightly improved in all 330 three combinations; the best case was adding $1 \mathrm{~m} /$ pixel fea331 tures, yielding a $0.4 \%$ gain in accuracy. However, the addi$3 з 2$ tional memory and computation time needed to obtain such a ${ }_{33 з}$ small accuracy improvement made us discard the multiresolu334 tion approach for $\mathrm{S} 4 \mathrm{~S}$ purposes.

\begin{tabular}{lrrr}
\hline Feature sets & Accuracy & Training & Inference \\
\hline LBP, H & $78.34 \%$ & $93 \mathrm{~s}$ & $22 \mathrm{~s}$ \\
LBP, H, F(0.25 m) & $\mathbf{9 4 . 8 5 \%}$ & $91 \mathrm{~s}$ & $28 \mathrm{~s}$ \\
LBP, H, F(0.50 m) & $93.94 \%$ & $94 \mathrm{~s}$ & $28 \mathrm{~s}$ \\
LBP, H, F(1 m) & $93.09 \%$ & $97 \mathrm{~s}$ & $28 \mathrm{~s}$ \\
LBP, H, F(2 m) & $91.71 \%$ & $94 \mathrm{~s}$ & $28 \mathrm{~s}$ \\
\hline LBP, H, F(0.25 m), F(0.5 m) & $95.14 \%$ & $134 \mathrm{~s}$ & $37 \mathrm{~s}$ \\
LBP, H, F(0.25 m), F(1 m) & $95.26 \%$ & $106 \mathrm{~s}$ & $36 \mathrm{~s}$ \\
LBP, H, F(0.25 m), F(2 m) & $95.14 \%$ & $103 \mathrm{~s}$ & $36 \mathrm{~s}$ \\
\hline
\end{tabular}

Table 2: Average accuracies using different sets of feature resolutions.

\section{6.2. Feature contributions}

${ }_{336}$ Figure 5 shows the relevance (as \% of accuracy loss, follow337 ing [28]) of the tested features on randomly selected samples ${ }_{338}$ of the training set (Figure 11). The height feature appears to

$3 з$ play an important role in accuracy. However, it does not de340 pend on the appearance of the patch containing the pixel but on ${ }_{341}$ its absolute location, and so the causal relation learned by RF 342 (e.g. snow and water confined to specific height ranges) might 343 be very specific to the chosen exemplars, and could generalize 344 poorly on different scenes. Slope orientation can also intro${ }_{345}$ duce bias in the results. In our selected training set, we were 346 not aware that bushes regions had been selected only from a 347 few orientations, and thus when classifying full terrains we ob348 tained incorrect results. Therefore, we decided to remove these 349 two features (height, slope orientation) in our final model, re350 sulting in 88 features. Notice also the contribution of the NDVI 351 feature, which is computed from the near-infrared channel. For 352 datasets lacking this channel, we could still use our pipeline 353 expecting an accuracy around $6 \%$ lower.

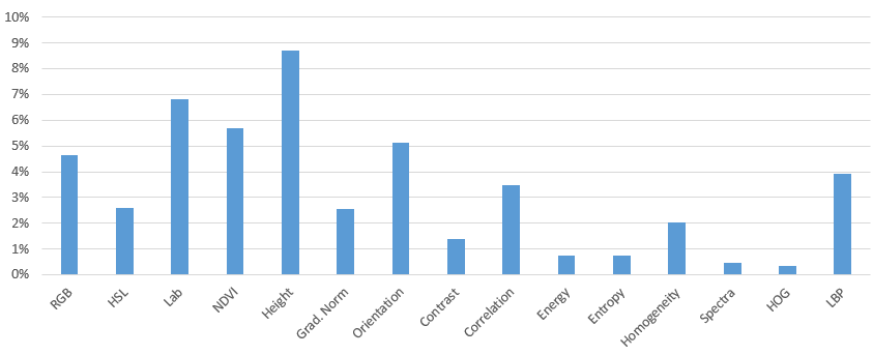

Figure 5: Accuracy loss after randomly permuting the samples of a certain feature group. Original accuracy $91.6 \%$.

\section{${ }_{354}$ 7. Training set sampling}

355 Unlike typical classification problems, with fixed classes and ${ }_{356}$ large training sets, we deal with dynamic classes and user357 provided exemplars. Given an exemplar image, one easy way 358 to construct a training set is to ask the user to select manually a 359 few homogeneous regions for each class. The system will then 360 properly sample a subset of the labeled pixels and use them as 361 training data. This option puts much less effort on the user than 362 asking for a completely-segmented exemplar.

${ }_{363}$ In this setup, we have to face three different problems:

- Non-representative neighborhoods: since training instances will be selected from the manually-identified homogeneous regions, the corresponding pixels are likely to have coherent neighborhoods that are not necessarily representative of arbitrary pixels (in contrast to selecting arbitrary pixels from a completely-segmented exemplar).

- Imbalanced data: in some exemplars, the ratio of the majority class (e.g. tree) to the minority class (e.g. scree) instances can be very large. This can be due to the predominance of some classes in the chosen exemplars, or to the fact that some objects (e.g. pathways) can be more difficult to select manually than others (e.g. forest). Therefore some degree of class imbalance is expected. 


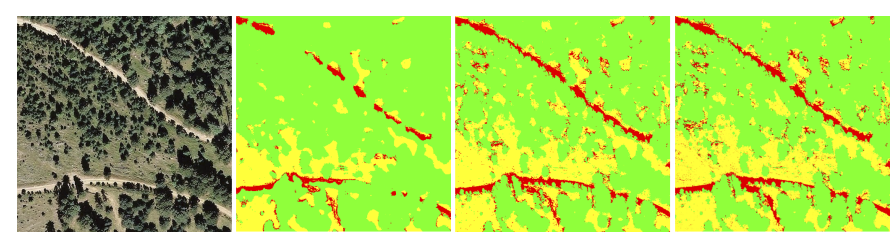

Figure 6: From left to right: part of the photograph, classification using homogeneous neighborhoods, classification using heterogeneous neighborhoods, and manual classification.

382 We will first address the non-representative neighborhood ${ }_{383}$ problem. We conducted an experiment to evaluate how large 384 was the effect of training with data coming from homogeneous 385 regions. We used again the well-separated Dataset Andorra, for 386 which a complete segmentation was available. Each pixel was ${ }_{387}$ considered to be homogeneous if all pixels within its $r \times r$ neigh388 borhood had the same class. Otherwise, the pixel was consid389 ered to be heterogeneous. Then, we built three training sets: 390 one with only homogeneous pixels, one with only heteroge391 neous pixels and one with $50 \%$ of each type. Each training 392 set was randomly undersampled to about $260 \mathrm{~K}$ training exam393 ples. We trained a $R F(D=15, N=50)$ for each training set and 394 measured the resulting accuracies on a validation set including 395 pixels from all across the image. Figure 6 compares the re396 sulting segmentations on a small part of the image. Averaged 397 accuracies are shown in Table 3.

\begin{tabular}{crrr}
\hline Window size & Homogeneous & Half and half & Heterogeneous \\
\hline$r=2$ & $92.9 \%$ & $\mathbf{9 5 . 2 \%}$ & $93.7 \%$ \\
$r=5$ & $89.7 \%$ & $\mathbf{9 5 . 1 \%}$ & $94.9 \%$ \\
$r=10$ & $86.8 \%$ & $94.9 \%$ & $\mathbf{9 5 . 1 \%}$ \\
$r=15$ & $84.6 \%$ & $94.9 \%$ & $\mathbf{9 5 . 1 \%}$ \\
\hline
\end{tabular}

Table 3: Average accuracies depending on the training set selection.

398 These results show the positive impact of including some ex399 amples near the (image-space) class boundaries. Both the het400 erogeneous and the $50 \%-50 \%$ training sets resulted in high ac401 curacy, since both include examples with representative neigh402 borhoods. However, if training examples are taken from ho403 mogeneous regions, we cannot guarantee representative neigh404 borhoods as long as user-selected region boundaries might not 405 match class boundaries. Fortunately, we found that reducing the 406 window size used for the homogeneity criterion (i.e. allowing 407 homogeneous pixels to get closer to class boundaries) leaded 408 to reasonable accuracy (above $90 \%$ in the experiment, see Ta409 ble 3). Since a complete pixel-wise segmentation of the exem${ }_{410}$ plars is a very time-consuming task, a good trade-off is achieved ${ }_{411}$ by asking users to select nearly-maximal homogeneous regions, 412 with its boundary near examples from other classes. Following ${ }_{413}$ this guideline, exemplars are still easy to label (with respect
${ }_{414}$ to a complete pixel-wise segmentation) while still resulting in 415 reasonably good classification accuracy.

${ }_{416}$ We now address the problems of imbalanced data and dis${ }_{417}$ similar class distributions. We analyzed the impact of different ${ }_{418}$ distributions on Andorra. The real distribution on this set is ${ }_{419} 74.5 \%$ tree, $13.7 \%$ grass and $11.8 \%$ ground. Figure 7 shows 420 the curve obtained when sampling different percentages of the ${ }_{421}$ class tree on the training set, and splitting the rest of the train422 ing set equally among grass and ground. As expected, the best 423 segmentation results are achieved when the training distribution 424 roughly matches the target distribution (vertical dashed red line 425 in Figure 7). However, we only observed small changes in the 426 overall accuracy even with completely erroneous distributions. ${ }_{427}$ Therefore we decided to give the user the opportunity to pro428 vide the expected class distribution on the type of images to 429 be classified (as rough per-class percentages). We sample the 430 training set according to the user-provided distribution, re-train ${ }_{431}$ the RF, and use it to segment the input image.

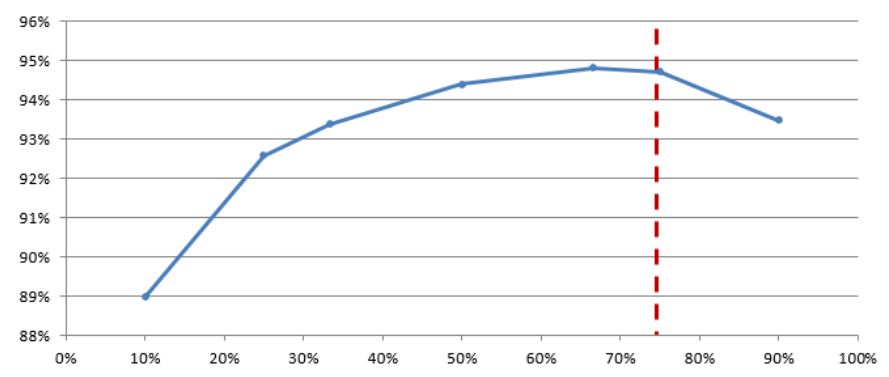

Figure 7: Accuracy obtained for different distributions of the training set samples.

\section{${ }_{432}$ 8. Smoothing class probabilities}

433 The output of our classifier is a set of class probabilities ${ }_{434}$ rather than just the class label. Therefore, users can apply post435 classification filtering techniques to correct noise or small errors 436 in the final labeling.

${ }_{437}$ Schindler [11] compares different smoothing methods that 438 can be used to produce a final labeling from class probabilities. ${ }_{439}$ Due to their speed and simplicity, we decided to implement the 440 three filtering methods he presents: majority voting, Gaussian ${ }_{441}$ filter and bilateral filter. Additionally, the user can provide, for ${ }_{442}$ each label, the minimum expected footprint $\sigma$ of the objects ${ }_{443}$ belonging to this class. For example, a single pixel $(0.25 \mathrm{~m}$ in ${ }_{444}$ our case) labeled as tree surrounded by non-tree pixels is very 445 likely to be erroneous, while a single grass pixel may be plausi446 ble. Figure 8 shows an example in which no filtering results in 447 a very noisy classification, while a bilateral filter over-smooths 448 some grass or bush regions. However, filtering with a differ${ }_{449}$ ent $\sigma$ on each probability map eliminates undesired single-pixel 450 trees while keeping small vegetation clusters. 


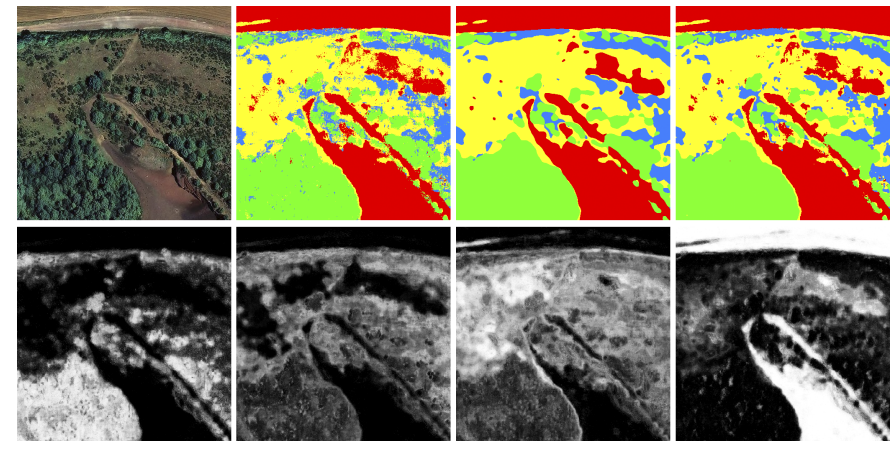

Figure 8: Top row: input image, classification output without filtering (MAP), bilateral filter with $\sigma=4$ pixels, bilateral filter with $\sigma_{\text {tree }}=4, \sigma_{\text {bush }}=2$, $\sigma_{\text {grass }}=1$ and $\sigma_{\text {ground }}=0.5$ pixels. Bottom row: probability maps of classes tree, bush, grass and ground.

\section{${ }_{451}$ 9. Cost-sensitive classification}

452 Recall that we aim at replacing pixels by synthetic detail in 453 the context of free-camera applications. Misclassified pixels are 454 likely to have a varying perceptual impact, depending on the $455 \mathrm{predicted} /$ true labels and on the specific detail synthesis tech456 niques that will be applied to the output labeled images. For 457 example, misclassifying terrain with any of the other vegeta${ }_{458}$ tion classes (tree, shrub, grass) is likely to have a higher visual 459 impact than e.g. confusing shrubs and trees.

Given the estimated class probabilities $\hat{p}_{k}(x)$ for a test pixel $x$, its final class $\hat{y}(x)$ is computed as the one minimizing the expected misclassification costs,

$$
\hat{y}(x)=\arg \min _{i=1, \ldots K} \sum_{j=1}^{K} c_{i \mid j} \hat{p}_{j}(x)
$$

${ }_{460}$ where $c_{i \mid j}$ is the user-defined cost of misclassifying as class $i$ ${ }_{461}$ a pixel whose true label is $j$. Without loss of generality [29], ${ }_{462}$ elements on the diagonal of the cost matrix $C=c_{i \mid j}$ can be 463 assumed to be 0 , and elements off-diagonal can be assumed to ${ }_{464}$ have a positive cost. Scaling $C$ by a positive constant does not 465 affect the optimal decision, so the minimum non-zero cost can 466 be assumed to be 1 . For $K=4$ classes, the cost matrix requires ${ }_{467}$ only 11 values to be provided by the user. In Section 10 we pro468 vide some insights on how to derive well-founded cost matrices 469 based on perceptual differences between pairs of classes.

\section{10. Results}

\section{10.1. Classification accuracy of human labelers}

472 We conducted a first experiment to analyze how humans clas473 sify regions in aerial images. The purpose was two-fold: to 474 obtain ground truth labels to test our algorithm with, and to 475 analyze the difficulty and resulting accuracy of human label476 ers. Since per-pixel classification is a long and tedious task, 477 we selected a total of 56 uniform regions from 8 datasets (Fig478 ure 9-top) representing a variety of forest and rural areas around ${ }_{479}$ Catalonia.
480 For each region, we asked users to choose between four la481 bels: tree, bush, grass and ground, as well as three additional la482 bels expressing doubts between two classes: tree or bush, bush ${ }_{483}$ or grass, grass or ground. During the test, one region at a time 484 was presented to the user as a blinking, semi-transparent over485 lay on top of the current aerial photograph. Users were able to 486 zoom in and out using the mouse wheel. Twenty-three users 487 (19 male, 4 female, ages 22-45, normal-sighted, most of them 488 familiar with computer games) participated in the experiment.

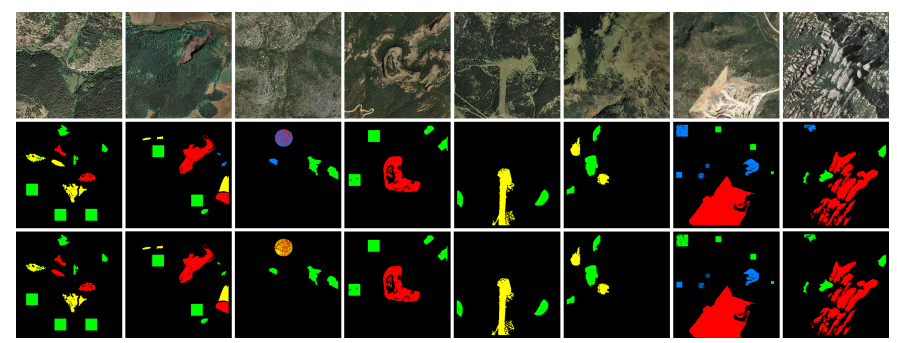

Figure 9: Datasets used in the first user study (top), ground-truth labels computed following [30] (center), majority class given by our per-pixel classification (bottom). The datasets shown are (from left to right): Andorra, Olot, Garraf, Rocacorb, Peguera, Setcases, Garraf-Quarry, Montserrat.

489 Ground-truth labels were not directly available, so we com490 puted and compared against the crowdsourced labels. One way 491 to obtain these labels is through majority voting, i.e. taking the 492 most voted label at each region. However, this strategy does 493 not account for how good each individual labeler is, nor the 494 intrinsic difficulty of labeling each particular region. Following 495 Whitehill et al. [30], we inferred the label of each region using a 496 probabilistic graphical model in which observed labels depend 497 on three causal factors: difficulty of the image, expertise of the 498 labeler, and the true label of the image. Using an Expectation499 Maximization approach, all three causal factors can be inferred 500 from the observed labels. For our special case in which a user 501 expressed doubt between two classes, we treated this as two 502 different observed labels for that region given by the same user.

Figure 9-middle shows the resulting ground truth labels using 504 the implementation provided in [31]. These labels will be used 505 in Section 10.2 to test our classification pipeline.

506 Alongside ground truth labels, we can also read from the 507 probabilistic model the region difficulty parameter $\beta$. White508 hill et al. [30] model image difficulty as $1 / \beta \in[0, \infty)$. When ${ }_{509} \beta$ is close to 0 , the image difficulty is very high and even the 510 most skilled users become just pure random labelers. Figure 10 511 shows the $\beta$ parameters averaged by input image (left) and by 512 ground truth label (right). Notice that difficulty estimates ex513 hibit large variance across classes and datasets. In particular, 514 bush regions are hard to classify, and consequently images that 515 contain regions similar to bushes are more prone to misclassifi516 cation errors.

517 Table 4 shows the resulting users' confusion matrix. Partici518 pants were relatively good at distinguish between high and low 519 vegetation (tree/grass), but bushes were easily confused with ${ }_{520}$ trees or grass. These confusion matrices provide a perceptual ${ }_{521}$ basis to measure how easy a label can be misclassified as an- 


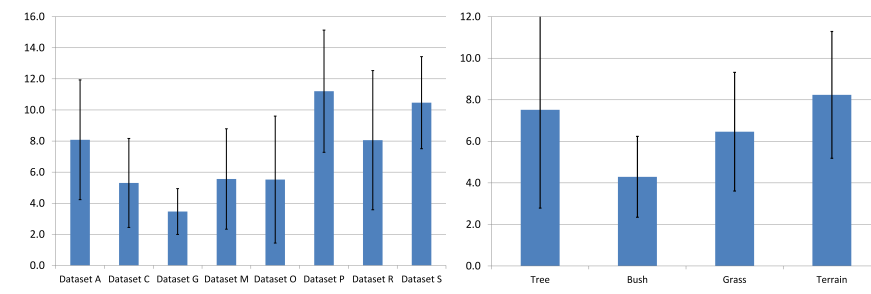

Figure 10: Average classification easiness for each dataset (left) and for each class (right). The lower the value, the higher the difficulty.

522 other label, and thus provide a solid foundation to build percep${ }_{523}$ tual cost matrices: in the context of S4S, the higher the confu524 sion rate between two classes, the lower should be the corre525 sponding misclassification cost.

\begin{tabular}{rrrrr}
\hline Class / Predicted & Tree & Bush & Grass & Ground \\
\hline Tree & $85.4 \%$ & $14.6 \%$ & $0.0 \%$ & $0.0 \%$ \\
\hline Bush & $11.1 \%$ & $63.9 \%$ & $23.7 \%$ & $1.3 \%$ \\
\hline Grass & $0.0 \%$ & $0.4 \%$ & $95.4 \%$ & $4.0 \%$ \\
\hline Ground & $0.0 \%$ & $0.2 \%$ & $8.3 \%$ & $91.5 \%$ \\
\hline
\end{tabular}

Table 4: Confusion matrix as percentages per label.

\section{10.2. Classifier accuracy vs human accuracy}

${ }_{527}$ The user study above shows that identifying tree, shrub, grass 528 and terrain classes on $25 \mathrm{~cm} /$ pixel aerial images is not an easy 529 task, and that users often express doubt or misclassify well530 delimited regions. This suggests that a manual segmentation ${ }_{531}$ from a single user is just a biased estimate of the ground truth. ${ }_{532}$ Therefore, we decided to conduct a follow-up study. We asked ${ }_{533}$ eight new users ( 7 male, 1 female, ages 22-32, normal-sighted, 534 all of them familiar with computer games) to classify the re535 gions in Figure 9, but this time only the four classes were avail${ }_{536}$ able to them, as they were not allowed to express doubt.

${ }_{537}$ The number of regions each user marked differently from the ${ }_{538} 23$-subject ground truth were between 5 and 10, with an average 539 of 7.5 incorrectly classified regions out of 56 (87\% accuracy).

${ }_{540}$ We compared this user accuracy with that of our classifier, ${ }_{541}$ keeping the majority class inside each of the regions to produce ${ }_{542}$ a unique label of the whole region. The classifier output showed ${ }_{543} 7$ misclassified regions (see Figure 9 bottom row), similar to the 544 expected error for human labelers. All but one of these error 545 regions correspond to what users perceived as ground truth bush 546 and were confused with trees or grass, a distinction that is also ${ }_{547}$ usually hard for humans as we explained in Section 10.1.

\section{10.3. Visual validation of complete segmentations}

${ }_{549}$ We used our classifier to perform full per-pixel classifica550 tions of different datasets. The first eight datasets consisted of a ${ }_{551} 3600 \times 3600$ aerial image (rgb and infrared), and the correspond552 ing digital elevation model. These datasets were segmented by 553 our algorithm into four classes: tree, bush, grass, and ground. ${ }_{554}$ The last three datasets were bigger $(16000 \times 16000$ pixels $)$ and 555 included four additional classes: scree, rock, water, and snow.
${ }_{556}$ The test hardware was a single PC equipped with an Intel ${ }_{557}$ Core i7 at $3.40 \mathrm{GHz}$ and $16 \mathrm{~GB}$ of RAM. We used OpenCV 558 and Matlab to extract the 88 features of each pixel, with a per559 formance of $36 \mathrm{~s} / \mathrm{Mpixel}$. Note that features only need to be 560 extracted once per image.

${ }_{561}$ The classifier, as well as the various tests presented in pre562 vious sections, were implemented using KNIME [32]. The ${ }_{563}$ training set was randomly sampled from a gallery of nearly564 maximal homogeneous regions that was easily segmented by 565 an expert (Figure 11), the proportion of samples of each class 566 being user-adjustable. These training images are not contained 567 in any of the datasets to classify. Training a random forest us568 ing $400 \mathrm{~K}$ samples took about $2 \mathrm{~min}$. Classification took around ${ }_{569} 21.5 \mathrm{~s} /$ Mpixel ( $5 \mathrm{~min} 45 \mathrm{~s}$ per $\mathrm{km}^{2}$ ).

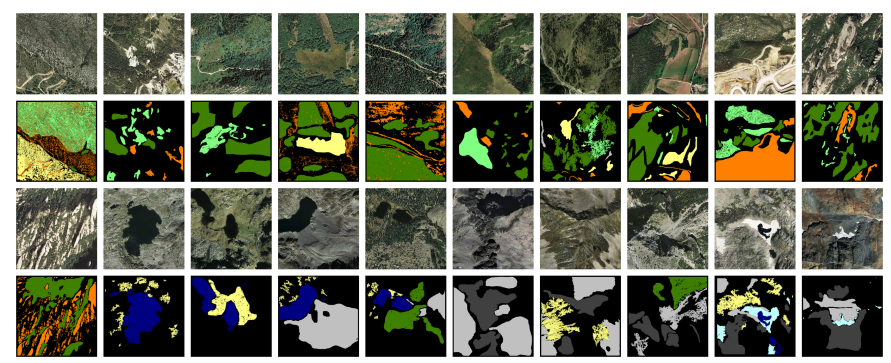

Figure 11: The 20 images used as training set with nearly-maximal homogeneous regions segmented. Classes: tree (dark green), bush (light green), grass (yellow), ground (orange), scree (light grey), rock (dark grey), water (blue), snow (cyan).

Figure 12 shows the 11 datasets as well as the output of 571 our classifier - as the most probable class - before applying 572 smoothing or cost matrix. These segmentations can be assessed 573 only visually since no ground-truth is available.

\section{10.4. Detail synthesis application}

575 The most relevant application of the proposed pipeline is to 576 synthesize terrain and vegetation detail depending on the per577 pixel labels. Here we show some sample images obtained by 578 a straightforward approach. Regions labeled as trees or shrubs 579 were covered by a blue noise pattern, each point representing 580 the location of a synthetic tree/shrub. The minimum distance 581 between generated points was determined by the size of the 582 synthetic vegetation models. Grass regions were enhanced by ${ }_{583}$ instantiating 3D models of grass and flowers. Fragments cov584 ered by water and snow were rendered using specific shaders. ${ }_{585}$ Terrain regions were detailed on-the-fly through displacement ${ }_{586}$ mapping shaders perturbing the original DTM according to ${ }_{587}$ Fractional Brownian Motion noise. Figures 13 and 1 show 588 some resulting images (see accompanying video).

\section{10.5. Detail synthesis validation}

590 We conducted a final user study to evaluate the quality of 591 our segmentation pipeline in the context of detail synthesis. We 592 selected a set of 30 viewpoints on a large DEM (Figure 12, last 593 column), and rendered the scene with procedurally-generated 594 detail (vegetation, rocks, water) placed according to either our 

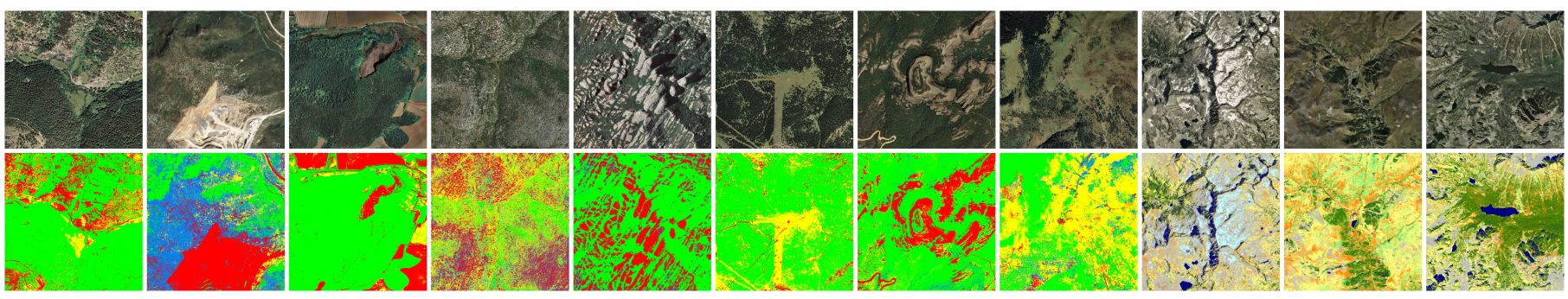

Figure 12: Classifier results for our 11 datasets. The first eight datasets were segmented into 4 classes (tree, bush, grass, ground) while the last three were segmented into 8 classes (tree, bush, grass, ground, scree, rock, water, snow). We use different color maps to highlight this. From left to right, the datasets are: Andorra, Garraf-Quarry, Olot, Garraf, Montserrat, Peguera, Rocacorb, Setcases, Besiberris, Nuria, Sant Maurici.
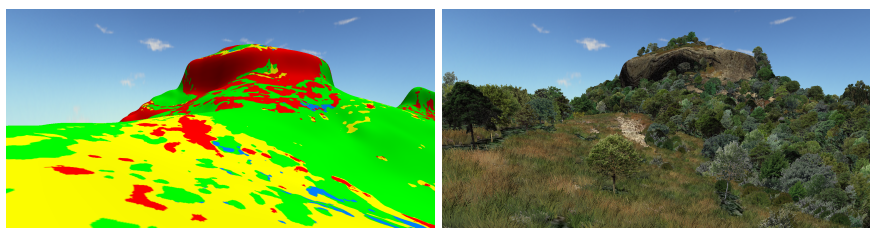

Figure 13: Example application: vegetation synthesis on terrain. Dataset Rocacorb is shown with the result of the classifier (left) and the rendered images with vegetation on the terrain (right).

595 segmented image (OURS) and an official $5 \mathrm{~m} /$ pixel land cover 596 map (LCM). The set of images used in the study can be found 597 in the supplementary material.

598 Our study consisted of a selection task, in which participants 599 were presented both images (OURS/LCM) and had to choose 600 which one showed a better placement of the synthetic elements, 601 and a scoring task, in which participants were shown one partic602 ular image at a time (either OURS or LCM) and had to indicate ${ }_{603}$ the plausibility of detail placement using a 4-point Likert scale. ${ }_{604}$ Both tasks started with a tutorial video and displayed instruc605 tions throughout the trials.

606 For the selection task, participants were shown 12 random 607 image tuples (from the set of 30), where each tuple consisted 608 of rendered images from the same viewpoint, using either our 609 segmented image or the LCM. Users were requested (forced 610 choice) to select the best image in terms of the placement of ${ }_{611}$ the added elements. A reference image showing just the DEM ${ }_{612}$ textured with the orthophoto was also shown. For all partici${ }_{613}$ pants, the order of the tuples was randomly chosen, along with 614 the order of the images within the tuple.

${ }_{615}$ For the scoring task, we randomly selected 12 images as in ${ }_{616}$ the first task, but now each image was shown separately rather 617 than in a tuple. One half of the images were detailed using ${ }_{618}$ our segmentation, the other half through LCM. Participants 619 were asked to assign a score $\{1,4\}$ based on how plausible they 620 thought the detailed elements were placed, again according to a 621 reference (no synthetic detail) image.

622 The study was deployed on a website, and participants (21, ${ }_{623}$ contacted through email) could complete the task remotely us624 ing the device of their choice. We applied Bayesian data anal625 ysis [33] using an experiment design in the same spirit of [34]. ${ }_{626}$ Reported results represent the posterior mean, and the confi-
${ }_{627}$ dence interval (CI) represents the range including $95 \%$ of the ${ }_{628}$ posterior probability.

${ }_{629}$ Concerning the selection task, we modeled the posterior ${ }_{630}$ probability of each method (OURS, LCM) being selected as ${ }_{631}$ the best as a Bernoulli random variable with a uniform Beta ${ }_{632}$ prior. As shown in Figure 14-left, our method was significantly ${ }_{633}$ more likely to be selected as the best in terms of detail place${ }_{634}$ ment, when comparing against LCM-based placement. Fig635 ure 15 shows also the posterior probability, but this time con${ }_{636}$ ditioned to the viewpoint type (aerial/ground); as expected, the ${ }_{637}$ advantage of OURS over LCM is higher for ground-level views 638 where element misplacement is more apparent and the lower 639 resolution of the LCM plays an important role. Nevertheless, 640 our segmentation outperformed LCM also for aerial views, pre${ }_{641}$ sumably because LCM involves manual user input and lacks 642 the fine-grained contours of our fully-automatic per-pixel clas643 sification.

${ }_{644}$ Regarding the scoring task, we computed how likely each 645 segmentation was to get the highest plausibility score in the 4${ }_{646}$ Likert scale. Figure 14-right shows that our method was signifi647 cantly more likely to get the highest score. Summarizing, these 648 results show that our segmentation pipeline is suitable for plau${ }_{649}$ sible detail synthesis and that it outperforms an official (expert650 assisted) land cover map.
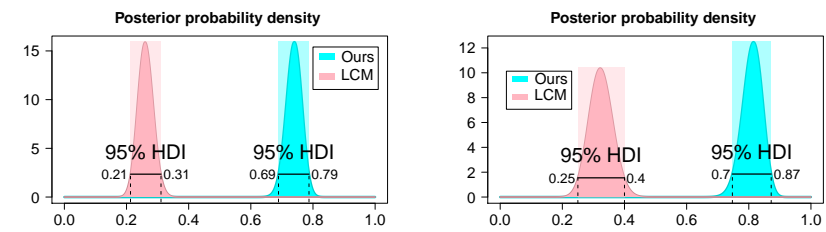

Figure 14: Left: Posterior probability $\mathrm{p}(\theta \mid D)$ of each method (OURS, LCM) of being chosen as the most plausible in a selection round. LCM: $26 \%$ (21\%$31 \%$ HDI); Ours: $74 \%$ (69\%-79\% HDI). Right: Posterior probability of each method (OURS, LCM) of getting the highest score in the scoring task. LCM: $32 \%$ (25\%-40\% HDI); Ours: $81 \%$ (75\%-87\% HDI). Shaded rectangles indicate the $95 \%$ Highest Density Interval (HDI).

\section{11. Fully Convolutional Neural Networks}

Recently, Deep Learning has been successfully applied to ${ }_{653}$ image segmentation using Fully Convolutional Neural Net- 

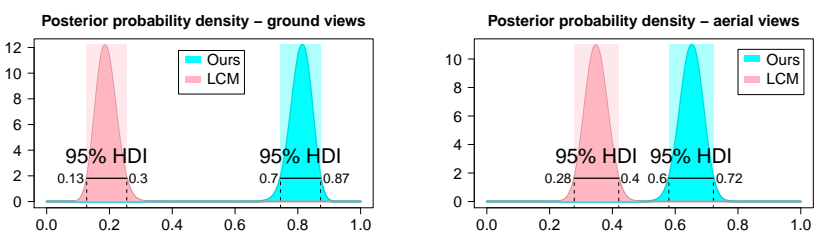

Figure 15: Posterior probability of winning a selection round, conditioned on the camera elevation: close-up views ( $1 \mathrm{~m}$ to $30 \mathrm{~m}$, left), and aerial views ( $90 \mathrm{~m}$ to $180 \mathrm{~m}$, right).

${ }_{654}$ works (FCN). In [35], Long et al. convert image classification 655 networks into per-pixel segmentation networks by turning the ${ }_{656}$ fully-connected layers into convolutions, thus being able to out${ }_{657}$ put classification maps from any input size. Since typical clas658 sification architectures apply several pooling layers that reduce 659 the size of the original image, the output classification map has 660 lower resolution. The FCN proposed by Long et al. performs ${ }_{661}$ upsampling as well as fusing information from previous lay${ }_{662}$ ers in order to output a per-pixel segmentation. They are able ${ }_{663}$ to reduce the maximum upsampling required from $32 \times$ to $8 \times$, ${ }_{664}$ but explain that fusing further layers to reduce the upsampling 665 only yields slight improvements at a larger cost. In order to 666 evaluate the use of FCNs in our pipeline, we used their imple667 mentation and trained weights of the network FCN-8s-atonce 668 using Caffe [36]. This network does an 8x upsampling and re${ }_{669}$ ported their best performance in the PASCAL VOC segmenta670 tion dataset.

671 First, we modified the last layer of the net to match our 672 classes, and retrained using our fully-segmented Dataset An673 dorrasplit as $75 \%$ training and $25 \%$ validation. After 10 train674 ing epochs ( $11 \mathrm{~min} 38 \mathrm{~s}$ ), the obtained accuracy was $89.26 \%$. ${ }_{675}$ Classifying a $1000 \times 1000$ image took $15.42 \mathrm{~s}$ in CPU, $0.84 \mathrm{~s}$ in ${ }_{676}$ GPU. Figure 16 shows the output of this network. Note that the 677 output is smooth, as the network is in fact learning an $8 \times$ up678 sampling of the per-pixel labeling in its last layers. Modifying 679 the network to avoid this upsampling was out of the scope of 680 our work.

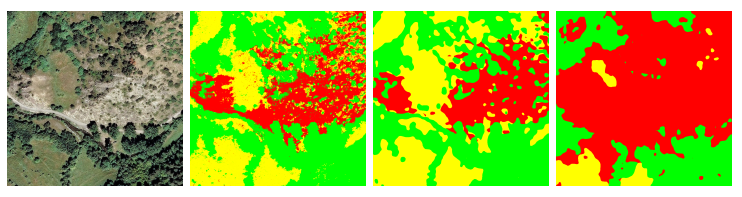

Figure 16: Part of Dataset Andorra. Left: aerial image, center-left: ground truth, center-right: classification using FCN-8s-atonce [35] with fully-classified training set, right: classification using FCN-8s-atonce [35] with nearlymaximal homogeneous regions training set.

${ }_{681}$ In the first test above, we trained the FCN using a complete 682 per-pixel segmented dataset. However, our goal is to allow the 683 user to train the classifier using a few homogeneous training ${ }_{684}$ regions, as in our experiments in Section 10. If we train the 685 net in this way - by letting the loss function ignore pixels out686 side training regions -, results are much worse as shown in Fig687 ure 16 right-most image, which shows the classification after
688100 training epochs ( $4 \mathrm{~h} 40 \mathrm{~min}$ ). This can be caused by the net 689 not being allowed to learn the shapes of boundaries between 690 classes. Even after a huge training time, results are still far 691 from an acceptable segmentation, effectively rendering FCNs 692 unusable for our S4S pipeline.

\section{${ }_{693}$ 12. Conclusions and future work}

In this paper we have presented a complete pipeline for seg695 menting aerial images into tree, shrub, grass and terrain classes. ${ }_{696}$ Segmented images can be used by external vegetation synthesis 697 algorithms to generate plausible vegetation on top of publicly698 available DTMs, enabling detailed close-up views of real sce699 narios in videogames and entertainment applications.

700 The pipeline we propose is practical in the sense that relies on 701 RGB+I orthorectified images which are commonly available. 702 All datasets used for the experiments were downloaded from 703 the public WMS of the Cartographic and Geological Institute of ${ }_{704}$ Catalonia (ICGC) ${ }^{1}$. Using hyperspectral images or LiDAR data 705 would clearly facilitate the segmentation, but at the expense of 706 severely restricting the applicability of our approach to selected 707 places.

708 The main issues arising when segmenting aerial images for 709 generating synthetic detail can be summarized as follows: the 710 classes (tree, shrub, grass and terrain are likely to be just a sub711 set of the required classes), the associated CPU/GPU rendering 712 techniques, and the visual impact of misclassification errors, are 713 all scenario-dependent. Considering the large amount of time 714 and effort required to obtain massive training sets, we have re715 stricted our attention to classification algorithms able to deal 716 with relatively small training sets.

717 Our experiments show that Random Forests provide an ex718 cellent option considering accuracy and performance both at 719 training and inference times. RF training times in the order of 720 a few minutes allow artists to experiment with different sets of ${ }_{721}$ classes (e.g. adding water or sand) and to add training examples 722 from new segmented regions as soon as they become available. 723 Fast inference times enable fast segmentation and data amplifi724 cation.

725 We have analyzed the contribution of standard color and tex726 ture features in the context of S4S. Adding features from down727 sampled images only yielded very slight accuracy improve728 ments. We did expect a higher impact, considering the fact that 729 human perception of vegetation types (at least on $25 \mathrm{~cm} /$ pixel 730 aerial images) seems to be affected by surrounding regions (dur731 ing the user study we observed that many users had to zoom in 732 and out multiple times to classify some regions). The analy${ }_{733}$ sis of feature contributions revealed the key role of NDVI, an 734 excellent discriminator of non-dry vegetation.

735 Regarding the impact of training set selection from manually 736 segmented images, we have shown that training examples from 737 homogeneous regions (the fastest manual labeling approach) 738 lead to suboptimal accuracies. These results should help to 739 guide human labelers to create effective training sets.

${ }^{1}$ http://geoserveis.icgc.cat/icc_mapesbase/wms/service? 
740 In our experiments with different datasets, our pipeline ${ }_{741}$ achieved an average $90 \%$ accuracy, which we take as a very 742 good result considering the low accuracy of individual users 743 when asked to classify manually these images.

744 As future work, we plan to enhance our segmentation ap745 proach with regression to estimate the height of vegetation; esti746 mated height values could replace the individual labels for tree, 747 shrub and grass classes. We also plan to extract features from ${ }_{748}$ LiDAR data, mainly to get massive training sets automatically 749 (rather than using it as input for the segmentation, due to less 750 availability of LiDAR data compared to aerial images). Tools ${ }_{751}$ for facilitating the manual segmentation of images tailored for 752 the chosen labels and adapted to $\mathrm{S} 4 \mathrm{~S}$ problems is also an inter753 esting venue for future work.

${ }_{754}$ Acknowledgements. This work has been partially funded by 755 the Spanish Ministry of Economy and Competitiveness and 756 FEDER under grants TIN2014-52211-C2-1-R and TIN2014757 56919-C3-2-R; and the Spanish Ministry of Education, Culture 758 and Sports PhD grants FPU13/01079 and FPU14/00725.

\section{${ }_{759}$ References}

[1] Ruiz LA, Fdez-Sarría A, Recio JA. Texture feature extraction for classification of remote sensing data using wavelet decomposition: a comparative study. In: International Archives of Photogrammetry and Remote Sensing; vol. 35. 2004, p. 1682-750.

2] dos Santos JA, Penatti OAB, da Silva Torres R. Evaluating the potential of texture and color descriptors for remote sensing image retrieval and classification. In: VISAPP (2). 2010, p. 203-8.

[3] Tokarczyk P, Montoya J, Schindler K. An evaluation of feature learning methods for high resolution image classification. ISPRS Annals of Photogrammetry, Remote Sensing and Spatial Information Sciences 2012;I3:389-94. doi:10.5194/isprsannals-I-3-389-2012.

[4] Penatti OAB, Nogueira K, dos Santos JA. Do deep features generalize from everyday objects to remote sensing and aerial scenes domains? In: Computer Vision and Pattern Recognition Workshops (CVPRW), 2015 IEEE Conference on. 2015, p. 44-51.

[5] Aksoy S. Spatial techniques for image classification. Image processing for remote sensing 2008;:225-48. sification. Geoscience and Remote Sensing, IEEE Transactions on 2014;52(1):439-51.

[7] Castelluccio M, Poggi G, Sansone C, Verdoliva L. Land use classification in remote sensing images by convolutional neural networks. CoRR 2015;abs/1508.00092.

8] Fröhlich B, Rodner E, Denzler J. Semantic segmentation with millions of features: Integrating multiple cues in a combined random forest approach. In: Lee K, Matsushita Y, Rehg J, Hu Z, editors. Computer Vision - ACCV 2012; vol. 7724 of Lecture Notes in Computer Science. Springer Berlin Heidelberg. ISBN 978-3-642-37330-5; 2013, p. 218-31.

[9] Fröhlich B, Bach E, Walde I, Hese S, Schmullius C, Denzler J. Land cover classification of satellite images using contextual information. ISPRS Annals of Photogrammetry, Remote Sensing and Spatial Information Sciences 2013;II-3/W1:1-6. doi:10.5194/ isprsannals-II-3-W1-1-2013.

10] Tokarczyk P, Wegner J, Walk S, Schindler K. Features, color spaces, and boosting: New insights on semantic classification of remote sensing images. Geoscience and Remote Sensing, IEEE Transactions on 2015;53(1):280-95

1] Schindler K. An overview and comparison of smooth labeling methods for land-cover classification. Geoscience and Remote Sensing, IEEE Transactions on 2012;50(11):4534-45.

12] Andújar C, Chica A, Vico M, Moya S, Brunet P. Inexpensive reconstruction and rendering of realistic roadside landscapes. Computer Graphics Forum 2014;33(6):101-17.
[6] Cheriyadat AM. Unsupervised feature learning for aerial scene clas-
803 [13] Shaw GA, Burke HhK. Spectral imaging for remote sensing. Lincoln Laboratory Journal 2003;14(1):3-28.

805 [14] Dachsbacher C. Interactive terrain rendering: towards realism with procedural models and graphics hardware. Ph.D. thesis; University of Erlangen-Nuremberg; 2006.

[15] Zhou H, Sun J, Turk G, Rehg JM. Terrain synthesis from digital elevation models. Visualization and Computer Graphics, IEEE Transactions on 2007;13(4):834-48.

[16] Génevaux JD, Galin E, Peytavie A, Guérin E, Briquet C, Grosbellet F, et al. Terrain modelling from feature primitives. Computer Graphics Forum 2015;34(6):198-210.

[17] Peytavie A, Galin E, Grosjean J, Merillou S. Arches: a framework for modeling complex terrains. Computer Graphics Forum 2009;28(2):45767.

[18] Guérin E, Digne J, Galin E, Peytavie A. Sparse representation of terrains for procedural modeling. Computer Graphics Forum (proceedings of Eurographics 2016) 2016;35(2):177-87. URL: http://dx . doi .org/10. 1111/cgf . 12821. doi:10.1111/cgf. 12821.

[19] Argudo O, Andujar C, Chica A, Guérin E, Digne J, Peytavie A, et al. Coherent multi-layer landscape synthesis. The Visual Computer 2017;33(68):1005-15.

[20] Argudo O, Chica A, Andujar C. Single-picture reconstruction and rendering of trees for plausible vegetation synthesis. Computers \& Graphics 2016;57:55 - 67 .

27 [21] Shah MA, Kontinnen J, Pattanaik S. Real-time rendering of realisticlooking grass. In: Proceedings of the 3rd international conference on Computer graphics and interactive techniques in Australasia and South East Asia. ACM; 2005, p. 77-82.

[22] Boulanger K, Pattanaik SN, Bouatouch K. Rendering grass in real time with dynamic lighting. IEEE Computer Graphics and Applications 2009;29(1):32-41.

[23] He H, Garcia EA. Learning from imbalanced data. IEEE Transactions on Knowledge and Data Engineering 2009;21(9):1263-84.

[24] Stockman G, Shapiro LG. Computer Vision. 1st ed.; Upper Saddle River, NJ, USA: Prentice Hall PTR; 2001. ISBN 0130307963.

38 [25] Pouli T, Cunningham DW, Reinhard E. A survey of image statistics relevant to computer graphics. Computer Graphics Forum 2011;30(6):176188 .

[26] Roettger S. Ndvi-based vegetation rendering. In: Proceedings of the Ninth IASTED International Conference on Computer Graphics and Imaging 2007. CGIM '07; 2007, p. 41-5.

[27] Ojala T, Pietikäinen M, Mäenpää T. Multiresolution gray-scale and rotation invariant texture classification with local binary patterns. IEEE Trans Pattern Anal Mach Intell 2002;24(7):971-87.

[28] Čehovin L, Bosnić Z. Empirical evaluation of feature selection methods in classification. Intelligent data analysis 2010;14(3):265-81.

[29] O'brien DB, Gupta MR, Gray RM. Cost-sensitive multi-class classification from probability estimates. In: Proceedings of the 25 th international conference on Machine learning. ACM; 2008, p. 712-9.

[30] Whitehill J, fan Wu T, Bergsma J, Movellan JR, Ruvolo PL. Whose vote should count more: Optimal integration of labels from labelers of unknown expertise. In: Bengio Y, Schuurmans D, Lafferty JD, Williams CKI, Culotta A, editors. Advances in Neural Information Processing Systems 22. Curran Associates, Inc.; 2009, p. 2035-43.

7 [31] Mineiro P. Modeling mechanical turk. http: //www.machinedlearnings. com/2011/01/ modeling-mechanical-turk.html; 2011.

[32] Berthold MR, Cebron N, Dill F, Gabriel TR, Kötter T, Meinl T, et al. KNIME: The Konstanz Information Miner. Berlin, Heidelberg: Springer Berlin Heidelberg. ISBN 978-3-540-78246-9; 2008, p. 319-26.

3 [33] Kruschke J. Doing Bayesian Data Analysis. 2nd ed.; Academic Press; 2014. ISBN 9780124058880.

865 [34] Gryka M, Terry M, Brostow GJ. Learning to remove soft shadows. ACM Transactions on Graphics 2015;34(5):153:1-153:15.

867 [35] Long J, Shelhamer E, Darrell T. Fully convolutional networks for semantic segmentation. In: The IEEE Conference on Computer Vision and Pattern Recognition (CVPR). 2015,.

[36] Jia Y, Shelhamer E, Donahue J, Karayev S, Long J, Girshick R, et al. Caffe: Convolutional architecture for fast feature embedding. arXiv:14085093 2014;. 